\title{
Gynaecological Screening for Cervical and Vulvar Malignancies in a Cohort of Systemic Sclerosis Patients: Our Experience and Review of the Literature
}

\author{
M. Colaci, ${ }^{1}$ D. Giuggioli, ${ }^{1}$ G. Cassone, ${ }^{1}$ C. Vacchi, ${ }^{1}$ F. Campomori, ${ }^{1}$ F. Boselli, ${ }^{2}$ \\ M. Sebastiani, ${ }^{1}$ A. Manfredi, ${ }^{1}$ and C. Ferri ${ }^{1}$ \\ ${ }^{1}$ Chair and Rheumatology Unit, Medical School, Azienda Ospedaliero-Universitaria, Policlinico di Modena, \\ University of Modena and Reggio Emilia, Via del Pozzo 71, 41100 Modena, Italy \\ ${ }^{2}$ Institute of Obstetrics and Gynecology, Oncology Prevention Unit, University of Modena and Reggio Emilia, Modena, Italy \\ Correspondence should be addressed to M. Colaci; michelecolaci@virgilio.it
}

Received 19 August 2015; Revised 24 September 2015; Accepted 30 September 2015

Academic Editor: James R. Seibold

Copyright (c) 2015 M. Colaci et al. This is an open access article distributed under the Creative Commons Attribution License, which permits unrestricted use, distribution, and reproduction in any medium, provided the original work is properly cited.

\begin{abstract}
Background. Increased incidence of cancer was frequently reported in scleroderma (SSc), but no association with gynaecological malignancies was described in literature. Objectives. To investigate gynaecological neoplasms in SSc patients. Methods. In this cross-sectional analysis, we evaluated 80 SSc patients, living in the same geographical area. We considered all patients undergoing gynaecological evaluation, including pap test as screening for cervical cancer, between January 2008 and December 2014. Results. $55(68.7 \%)$ patients were negative and $20(25 \%)$ presented inflammatory alterations, while cancer or precancerous lesions were found in $5(6.2 \%)$ cases ( 2 showed cervical cancer (one of them in situ), 1 vulvar melanoma, 1 vulvar intraepithelial neoplasia, and 1 endocervical polyp with immature squamous metaplasia). The frequency of cervical cancer in our series seems higher in comparison to the incidence registered in the same geographical area. The presence of atypical cytological findings correlated with anti-Scl70 autoantibodies ( $p=0.022)$; moreover, the patients with these alterations tended to be older (median 65, range 46-67), if compared to the whole series $(p=0.052)$. Conclusions. A relatively high frequency of gynaecological malignancies was found in our SSc series. In general, gynaecological evaluation for SSc women needs to be included in the routine patients' surveillance.
\end{abstract}

\section{Introduction}

Increased incidence of malignancies was widely reported in systemic sclerosis (SSc) [1]. In particular, a strong association with lung cancer and, less frequently, with haematological neoplasms was described [2]. As for other autoimmune diseases, it is not surprising that the chronic inflammation and the abnormal tissue reparative processes increase the risk of malignancy, particularly in target organs such as sclerodermic lungs. Nonetheless, a relation with breast cancer was suggested, even if it remains controversial, since only a few epidemiological studies found it [3]. By contrast, an association with the cancer of the uterine cervix was not reported in literature, considering that cervical uterus malignancy is one of the most frequent cancers in women worldwide [4]. Because of its high frequency, public health programs of screening for cervical cancer have been established in several countries, in order to identify precancer lesions or to treat the disease at an earlier stage [5]; the conventional cytological Papanicolaou test (pap test) is recommended every 3 years for all women between 21 and 65 years old [6].

The aim of the present study is to investigate the results of pap test in a cohort of SSc women, who underwent the screening for cervical cancer.

\section{Patients and Methods}

In this cross-sectional analysis, we included all SSc patients who underwent gynaecological screening for malignancies, including pap test, during the period between January 1, 2008, and December 31, 2014. Namely, we evaluated 80 women (mean age $51.2 \pm 12$ years and disease duration $7.9 \pm 5.8$ years at the end of the study), referring to our University-Based 
TABLE 1: Clinical features and pap test screening findings in 80 SSc patients.

\begin{tabular}{|c|c|c|c|c|c|c|}
\hline & \multirow{2}{*}{$\begin{array}{l}\text { SSc series } \\
(n=80)\end{array}$} & \multirow{2}{*}{$\begin{array}{c}\text { Normal } \\
\text { A }(n=55)\end{array}$} & \multirow{2}{*}{$\begin{array}{l}\text { Inflammation } \\
\text { B }(n=20)\end{array}$} & \multirow{2}{*}{$\begin{array}{c}\text { Atypical cells/cancer } \\
\text { C }(n=5)\end{array}$} & \multicolumn{2}{|c|}{$p$ values } \\
\hline & & & & & (A versus $\mathrm{C})$ & $(A+B$ versus $C)$ \\
\hline Age at onset & $51.2 \pm 12$ & $51.2 \pm 12$ & $51.2 \pm 12$ & $65(46-67)$ & 0.052 & 0.06 \\
\hline SSc duration & $7.9 \pm 5.8$ & $8 \pm 5.8$ & $8 \pm 5.8$ & $6(1-13)$ & 0.45 & 0.45 \\
\hline \multicolumn{7}{|l|}{ Skin subsets } \\
\hline Limited & $72(90 \%)$ & $50(90.9 \%)$ & $18(90 \%)$ & $4(80 \%)$ & 0.42 & 0.42 \\
\hline Diffuse & $8(10 \%)$ & $5(9 \%)$ & $2(10 \%)$ & $1(20 \%)$ & 0.42 & 0.42 \\
\hline \multicolumn{7}{|l|}{ Serology } \\
\hline Anti-Scl70 & $23(28.7 \%)$ & $17(30.9 \%)$ & $2(10 \%)$ & $4(80 \%)$ & 0.046 & 0.022 \\
\hline ACA & $37(46.2 \%)$ & $28(50.9 \%)$ & $8(40 \%)$ & $1(20 \%)$ & 0.35 & 0.37 \\
\hline ANoA & $14(17.5 \%)$ & $6(10.9 \%)$ & $8(40 \%)$ & 0 & 1 & 0.58 \\
\hline Digital ulcers & $29(36.2 \%)$ & $21(38.2 \%)$ & $7(35 \%)$ & $1(20 \%)$ & 0.64 & 0.65 \\
\hline Interstitial lung disease & $30(37.5 \%)$ & $22(40 \%)$ & $8(40 \%)$ & 0 & 0.15 & 0.15 \\
\hline $\mathrm{FVC}<70 \%$ & $4(5 \%)$ & $3(5.4 \%)$ & 0 & $1(20 \%)$ & 0.30 & 0.23 \\
\hline DLCO $<70 \%$ & $46(57.5 \%)$ & $32(58.2 \%)$ & $12(60 \%)$ & $2(40 \%)$ & 0.65 & 0.65 \\
\hline $\mathrm{PAH}$ & 0 & & & & & \\
\hline History of smoking & $27(33.7 \%)$ & $16(29.1 \%)$ & $9(45 \%)$ & $2(40 \%)$ & 0.63 & 1 \\
\hline History of immunosuppressant & $5(6.2 \%)$ & $4(7.3 \%)$ & $1(5 \%)$ & 0 & 1 & 1 \\
\hline \multicolumn{7}{|l|}{ Pap test } \\
\hline Negative & $55(68.7 \%)$ & & & & & \\
\hline Inflammatory alterations & $20(25 \%)$ & & & & & \\
\hline Atypical cells/cancer & $5(6.2 \%)$ & & & & & \\
\hline
\end{tabular}

Rheumatology Centre, living in the same geographical area of the province of Modena (Northern Italy). In all cases the diagnosis of SSc was made by trained rheumatologists; anyway, all patients fulfilled the 2013 ACR/EULAR classification criteria for SSc [7].

Clinical records of epidemiological, clinical, serological, and instrumental data of all SSc patients along with analytic reports of the gynaecological evaluation were available for the study. Epidemiological data on cervical cancer were available online from Tumour Registry of the Province of Modena [8].

Statistical analysis was performed in order to investigate the possible associations between SSc parameters and cytological findings at pap test. Values are given as mean \pm $\mathrm{SD}$ for normally distributed variables, or as median (range) for not normally distributed variables. Group values and proportions were compared by univariate analysis of variance (ANOVA) and Fisher's exact test, respectively. The significant level adopted was $<5 \%$.

\section{Results}

Eighty patients presented at least one cytological evaluation of the cervix at the end of the study period. At pap test evaluations, $55(68.7 \%)$ patients were negative and 20 (25\%) presented inflammatory alterations consistent with chronic cervicitis, while atypical cells related to cervical cancer were found in $2(2.5 \%)$ cases, namely, a carcinoma in situ (CIN III) in one and invasive malignancy in the other one. In the remaining 3 patients the histological evaluation revealed a vulvar melanoma, a vulvar intraepithelial neoplasia (VIN III), and an endocervical polyp with immature squamous metaplasia, respectively. Overall, 5/80 (6.5\%) SSc patients showed precancerous/cancerous lesions of the cervix or external genital organs (Table 1).

The age of our SSc patients with neoplastic lesions was 65 years (range: 46-67). Data from the Cancer Registry of our province [8] show that the higher incidence of cervical cancer regards the age groups 30-44 and 45-59. Therefore, our SSc patients with cancer were older than what we expect based on our reference population.

The frequency of cervical cancer in our series (2 cases) seems to be higher than expected, considering the incidence registered in the same geographical area and in the same time period (standardized rate 8.0 cases, 95\% CI 5.2-10.7, out of 100,000 inhabitant-years) [8]. In fact, in the province of Modena, the cumulative risk to develop a cervical cancer during the entire life ( $0-74$ years) is $0.6 \%$, with a median age at diagnosis of 51 years [8]; thus, in our series of 80 patients followed up for $7.9 \pm 5.8$ years, we should expect less than 1 case ( 0.48 cases) of malignancy of the cervix. However, both the limited SSc series evaluated and the low number of cervical cancers observed did not permit obtaining a standardized incidence ratio (observed/expected events, SIR) with statistical significance.

In addition, the presence of atypical cytological findings correlated with serum anti-Scl70 autoantibodies (4/5 versus $19 / 75 ; p=0.022$ ), while patients with these alterations tended to be older (median 65, range 46-67), if compared to the whole series $(p=0.052)$. No statistical associations with skin or visceral SSc manifestations, smoking history, or treatment with immunosuppressors were found. 
TABLE 2: Cervical or vulvar cancers in SSc patients: review of the literature.

\begin{tabular}{|c|c|c|c|c|c|}
\hline First author, year [ref.] & Country & Type of study & patient-years $^{1}$ (pts/follow-up) & Cervical cancer & Vulvar cancer \\
\hline Duncan, 1979 [9] & Mayo Clinic, USA & Cohort study & $\left(2,141 /\right.$ n.d. $\left.{ }^{2}\right)$ & 10 & 1 \\
\hline Lee, 1983 [10] & Canada & Cohort study & $(95 / 7.3 \pm 6.6)$ & 2 & 0 \\
\hline Kyndt, 1997 [11] & France & Cohort study & $(123 /$ median 4$)$ & 1 & 0 \\
\hline Hill, 2003 [12] & South Australia & Registry-based & $(441 / 6.1 \pm 2.8)$ & 5 & 0 \\
\hline Chatterjee, 2005 [13] & Detroit area, USA & Registry-based & 4,908 & 3 (2 in situ) & 0 \\
\hline Derk, 2006 [14] & Pennsylvania, USA & Cohort study & 3,775 & 4 & 1 \\
\hline Olesen, 2010 [15] & Denmark & Registry-based & 16,003 & 9 & 0 \\
\hline Airo', 2011 [16] & Italy & Cohort study & 4,041 & 1 & 1 \\
\hline Belloli, 2011 [17] & Italy & Cohort study & $\left(112 /\right.$ n.d. $\left.^{3}\right)$ & $3^{4}$ & 0 \\
\hline Szekanecz, 2012 [18] & Hungary & Cohort study & (218/n.d.) & 1 & 0 \\
\hline Moinzadeh, $2014[19]^{5}$ & United Kingdom & Cohort study & $(2,177 /$ median 12.8$)$ & \multicolumn{2}{|c|}{17 "gynaecological" cases } \\
\hline Shah, $2015[20]^{6}$ & John Hopkins H, USA & Cross-sectional & $(1,044 / 1990-2012)$ & 3 & 1 \\
\hline Present series & Italy & Cohort study & 560 & 2 (1 in situ) & 2 (1 in situ) \\
\hline
\end{tabular}

(1) When the number of patient-years is not reported, number of patients (pts) and number of years of follow-up are indicated in parenthesis. (2) Authors reviewed histories of SSc patients seen between 1959 and 1975. (3) Authors indicated that the follow-up was approximately from 2004 to November 2009. (4) Uterine cancers. n.d.: not done. (5) This study considered the database of SSc patients followed at the Centre for Rheumatology and Connective Tissue Diseases of the Royal Free Hospital, London. The "gynaecological" cases of cancer were not better defined, and were not classified either before or after SSc onset. (6) This study evaluated the database of SSc patients observed at the John Hopkins Scleroderma Centre, Baltimore, in the period from 1990 to 2012 . Other 5 cases of cancer of the endometrium were cited.

All SSc series studied included male patients, except our cohort.

In our study, 2 cases of cervical cancer and 2 cases of vulvar cancers were found, as reported; the further case who presented an endocervical polyp with immature squamous metaplasia is not indicated.

\section{Review of the Literature}

To the best of our knowledge, no other studies in literature focused on cervical or vulvar cancer in SSc. Nonetheless, sporadic cases of these types of tumours are frequently cited in the list of the malignancies found during registry-based or cohort studies (Table 2) [9-18].

In a study regarding a large SSc series [9] seen at the Mayo Clinic between 1959 and 1975, 10 cases of cervical cancer were reported; curiously, in 5 cases the diagnosis of the tumour and the diagnosis of SSc were made in the same year, suggesting possible paraneoplastic cases. One case of vulvar carcinoma was also reported.

In a population-based cohort of 441 SSc patients from South Australia [12], 5 cervical cancers were signalled out of 90 cases of neoplasms registered. However, authors did not evaluate this finding by statistical analysis because of the small number of patients developing this malignancy. In another registry-based study on 538 SSc patients from the Detroit metropolitan area [13], 3 women with cervical cancer were found, 1 invasive and 2 in situ, in the absence of significantly increased incidence compared to general population. In a study evaluating a large series of 769 SSc patients prospectively followed up between 1987 and 2002 for the development of cancer [14], cervical cancer was recorded in 4/62 cases of malignancy observed after SSc diagnosis; one tumour of the vulva was also noticed. Again, the incidence of cervical-vulvar cancer observed in this patients series $(5 / 769,0.6 \%)$ was quite comparable to that reported in general population. Consistently, in the Danish nationwide population-based cohort study by Olesen et al. [15], among 162 cases of cancer in 1,586 female SSc patients, 9 cases of cervical cancer were observed (SIR 1.5, 95\% CI 0.7-2.8).
No cases of cervical cancer were registered in 2 large cohort studies in Japan [21] and Taiwan [22].

On the contrary, data of $218 \mathrm{SSc}$ patients followed up in a Hungarian University-Based Centre [18] evidenced 10 patients with malignancies; one of them was a 53-yearold woman with cervical cancer. Authors indicated that the SIR versus general population was 37.0 , but the interval of confidence was not calculated, so that the finding cannot be reliable.

Two recent studies $[19,20]$ on large SSc cohorts from the databases of highly specialised centres identified few cases of gynaecological cancers; however, these studies were designed for other purposes than the analysis of the incidence of the malignancies.

In 2009, Bernatsky et al. [23] investigated the history of an abnormal pap test by self-administrated questionnaire in a Canadian multicentre cohort of 320 SSc women. The lifetime prevalence of an abnormal pap test was $25.4 \%$, compared to $13.8 \%$ reported in the Canadian general population; moreover, a significant positive association with diffuse skin subset, smoking history, and younger age was noted. Even if a detection bias could influence these findings (SSc patients may be more likely to be aware of comorbidities than general population), authors concluded that it seems prudent to ensure adequate attention to cervical cancer screening for SSc women.

\section{Discussion}

We evidenced an apparently high frequency of cancerous lesions of the cervix or the vulva in our cohort of SSc patients. The increase of the incidence of cervical/vulvar cancer in SSc 
is not reported by the world literature; however, the present study is the first to specifically investigate the incidence of gynaecological (cervical and vulvar) tumours in patients with SSc, while the other studies found in literature (Table 2) are focused on research of every type of tumours (mainly infiltrating and life-threatening cancers); nonetheless, they are characterized by heterogeneous designs and are based on different population subsets. Therefore, it could be assumed that in SSc women the appearance of cervical or vulvar cancers, particularly in situ lesions (i.e., CIN and VIN III), could be underestimated in large cohort or registry studies. In fact, in our series, $3 / 5$ SSc patients with abnormal cytological tests did not present cervical cancer, but other pathological conditions that were generally excluded from the studies cited above, because of their low frequency.

Given these considerations, even if the cervical cancer, as specific clinical entity, may be considered as frequent as in the general population, periodic gynaecological screening for (pre)cancerous lesions of female genital organs should be carried out, since their occurrence might be not so uncommon in the course of SSc.

Pap smear abnormalities were also searched in other autoimmune disorders: in a case-control study [24] on 118 women affected mainly by systemic lupus erythematosus and rheumatoid arthritis, the frequency of abnormal pap tests was significantly higher in the patients ( $8.1 \%$ and $9.3 \%$, resp.) compared to controls (1.7\%); authors concluded that it might be an association between the autoimmune diseases and occurrence of (pre)cancer lesions of the cervix.

The main known trigger of cervical cancer is the human papillomavirus (HPV); in particular, a few specific virus genotypes are considered carcinogenetic [25]. Consequently, an increased risk of cervical cancer in SSc women, if confirmed, might indicate an increased infection/persistence of $\mathrm{HPV}$ in scleroderma patients. In this regard, no consistent data may be found in literature. Indeed, only one study [26] investigated the presence HPV infection and/or dysplasia in a small cohort of $31 \mathrm{SSc}$ women; comparable frequencies of the virus versus healthy controls were noticed, but infection by 2 or more HPV types was 2 times more frequent in SSc patients. The possibility of a link between SSc and HPV needs to be deeply studied.

Finally, the possibility of a selection bias with the consequent risk to overestimate cervical cancer incidence cannot be excluded. However, our data on pap test regarded less than 2-thirds of the SSc women population from province of Modena. Yet, considering the findings of the Italian National Centre of Epidemiology, Surveillance and Health Promotion in the "Passi 2012" study [27], more than 75\% of the women interviewed affirmed that they underwent a pap test in the last 3 years. This discrepancy points out to the possible risk that SSc patients, who are affected by a chronic disease with necessity to undergo a number of clinical and instrumental exams, could overlook other medical evaluations (such as the pap test), considered erroneously not so necessary for their peculiar health condition. Nonetheless, also family physicians might undervalue the screening exams recommended for general population for their highly "medicalised" female patients. Thus, even though an increased incidence of cervical cancer cannot be unequivocally affirmed by our study, it should be remembered to pay attention also to this medical aspect in SSc women, in order to ensure the best medical care, even if not directly related to scleroderma.

\section{Conclusions}

In our SSc patients' series, we found a relatively high frequency of cancerous lesions of the cervix by means of pap test. A significant association between gynaecological malignancies and anti-Scl70 autoantibodies was also found. These preliminary findings need to be verified in larger controlled epidemiological studies. In general, periodic gynaecological screening for SSc patients is truly suggested.

\section{Conflict of Interests}

All authors have no conflict of interests to declare.

\section{References}

[1] A. Onishi, D. Sugiyama, S. Kumagai, and A. Morinobu, "Cancer incidence in systemic sclerosis: meta-analysis of populationbased cohort studies," Arthritis and Rheumatism, vol. 65, no. 7, pp. 1913-1921, 2013.

[2] M. Bonifazi, I. Tramacere, G. Pomponio et al., "Systemic sclerosis (scleroderma) and cancer risk: systematic review and metaanalysis of observational studies," Rheumatology, vol. 52, no. 1, Article ID kes303, pp. 143-154, 2013.

[3] M. Colaci, D. Giuggioli, C. Vacchi et al., "Breast cancer in systemic sclerosis: results of a cross-linkage of an Italian Rheumatologic Center and a population-based Cancer Registry and review of the literature," Autoimmunity Reviews, vol. 13, no. 2, pp. 132-137, 2014.

[4] J. O. Parkhurst and M. Vulimiri, "Cervical cancer and the global health agenda: insights from multiple policy-analysis frameworks," Global Public Health, vol. 8, no. 10, pp. 1093-1108, 2013.

[5] L. Peirson, D. Fitzpatrick-Lewis, D. Ciliska, and R. Warren, "Screening for cervical cancer: a systematic review and metaanalysis," Systematic Reviews, vol. 2, article 35, 2013.

[6] Committee on Practice Bulletins-Gynecology, "ACOG practice bulletin no. 131: screening for cervical cancer," Obstetrics \& Gynecology, vol. 120, no. 5, pp. 1222-1238, 2012.

[7] H. Alhajeri, M. Hudson, M. Fritzler et al., "2013 American College of Rheumatology/European League against rheumatism classification criteria for systemic sclerosis outperform the 1980 criteria: data from the Canadian Scleroderma Research Group," Arthritis Care \& Research, vol. 67, no. 4, pp. 582-587, 2015.

[8] April 2015, http://www.rtm.unimo.it/pub2011.html.

[9] S. C. Duncan and R. K. Winkelmann, "Cancer and scleroderma," The Journal of the American Medical Association, vol. 115 , no. 8, pp. 950-955, 1979.

[10] P. Lee, C. Alderdice, S. Wilkinson, E. C. Keystone, M. B. Urowitz, and D. D. Gladman, "Malignancy in progressive systemic sclerosis-association with breast carcinoma," The Journal of Rheumatology, vol. 10, no. 4, pp. 665-666, 1983.

[11] X. Kyndt, M. Hebbar, V. Queyrel, E. Hachulla, P. Y. Hatron, and B. Devulder, "Sclérodermie systémique et cancer. Recherche de 
facteurs prédictifs de cancer chez 123 patients sclérodermiques," La Revue de Médecine Interne, vol. 18, no. 7, pp. 528-532, 1997.

[12] C. L. Hill, A.-M. Nguyen, D. Roder, and P. Roberts-Thomson, "Risk of cancer in patients with scleroderma: a population based cohort study," Annals of the Rheumatic Diseases, vol. 62, no. 8, pp. 728-731, 2003.

[13] S. Chatterjee, G. W. Dombi, R. K. Severson, and M. D. Mayes, "Risk of malignancy in scleroderma: a population-based cohort study," Arthritis and Rheumatism, vol. 52, no. 8, pp. 2415-2424, 2005.

[14] C. T. Derk, M. Rasheed, C. M. Artlett, and S. A. Jimenez, "A cohort study of cancer incidence in systemic sclerosis," Journal of Rheumatology, vol. 33, no. 6, pp. 1113-1116, 2006.

[15] A. B. Olesen, C. Sværke, D. K. Farkas, and H. T. Sørensen, "Systemic sclerosis and the risk of cancer: a nationwide populationbased cohort study," British Journal of Dermatology, vol. 163, no. 4, pp. 800-806, 2010.

[16] P. Airo', A. Ceribelli, I. Cavazzana, M. Taraborelli, S. Zingarelli, and F. Franceschini, "Malignancies in Italian patients with systemic sclerosis positive for anti-RNA polymerase III antibodies," The Journal of Rheumatology, vol. 38, no. 7, pp.13291334, 2011.

[17] L. Belloli, N. Carlo-Stella, N. Ughi, and B. Marasini, "Cancer in Italian patients with systemic sclerosis," European Journal of Inflammation, vol. 9, no. 2, pp. 199-203, 2011.

[18] É. Szekanecz, S. Szamosi, Á. Horváth et al., "Malignancies associated with systemic sclerosis," Autoimmunity Reviews, vol. 11, no. 12, pp. 852-855, 2012.

[19] P. Moinzadeh, C. Fonseca, M. Hellmich et al., "Association of anti-RNA polymerase III autoantibodies and cancer in scleroderma," Arthritis Research and Therapy, vol. 16, article R53, 2014.

[20] A. A. Shah, L. K. Hummers, L. Casciola-Rosen, K. Visvanathan, A. Rosen, and F. M. Wigley, "Examination of autoantibody status and clinical features associated with cancer risk and cancer-associated scleroderma," Arthritis \& Rheumatology, vol. 67, no. 4, pp. 1053-1061, 2015.

[21] A. Hashimoto, Y. Arinuma, T. Nagai et al., "Incidence and the risk factor of malignancy in Japanese patients with systemic sclerosis," Internal Medicine, vol. 51, no. 13, pp. 1683-1688, 2012.

[22] C.-F. Kuo, S.-F. Luo, K.-H. Yu et al., "Cancer risk among patients with systemic sclerosis: a nationwide population study in Taiwan," Scandinavian Journal of Rheumatology, vol. 41, no. 1, pp. 44-49, 2012.

[23] S. Bernatsky, M. Hudson, J. Pope et al., "Reports of abnormal cervical cancer screening tests in systemic sclerosis," Rheumatology, vol. 48, no. 2, pp. 149-151, 2009.

[24] H. Esmaeili and K. Ghahremanzadeh, "Association of Pap smear abnormalities with autoimmune disorders," Pakistan Journal of Biological Sciences, vol. 14, no. 10, pp. 600-604, 2011.

[25] M. Schiffman, G. Clifford, and F. M. Buonaguro, "Classification of weakly carcinogenic human papillomavirus types: addressing the limits of epidemiology at the borderline," Infectious Agents and Cancer, vol. 4, no. 1, article 8, 2009.

[26] M. Martin, C. Mougin, J. L. Prétet et al., "Screening of human papillomavirus infection in women with systemic sclerosis," Clinical and Experimental Rheumatology, vol. 32, 6, supplement 86, pp. S145-S148, 2014.

[27] 2015, http://www.epicentro.iss.it/passi/rapporto2012/ScreeningCervicale.asp. 


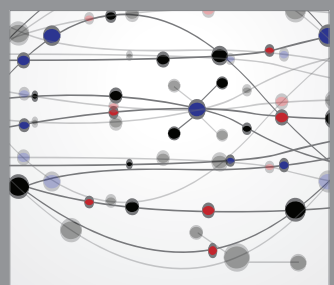

The Scientific World Journal
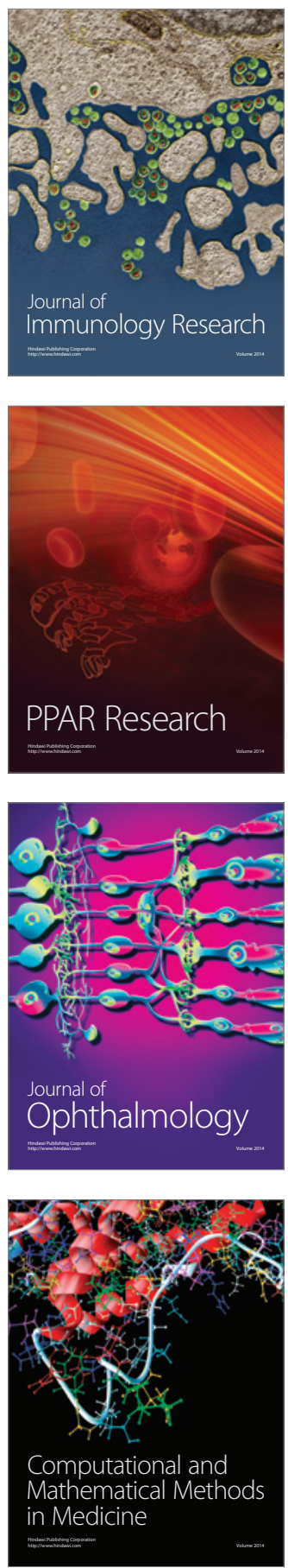

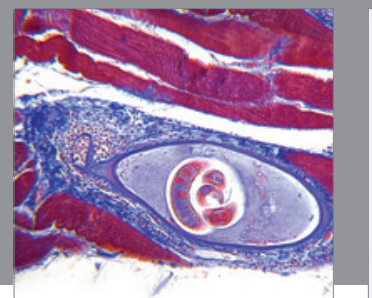

Gastroenterology

Research and Practice
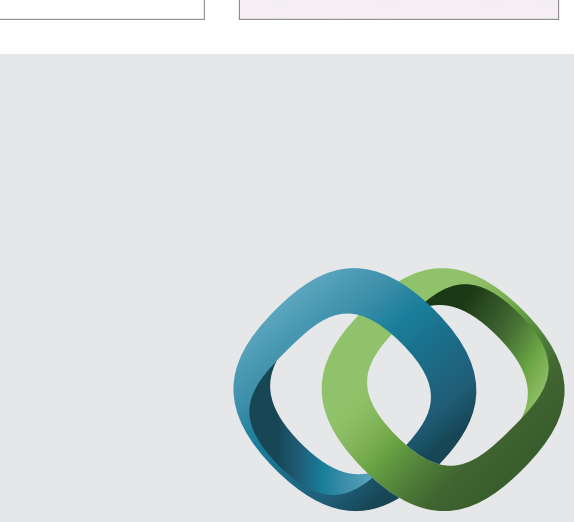

\section{Hindawi}

Submit your manuscripts at

http://www.hindawi.com
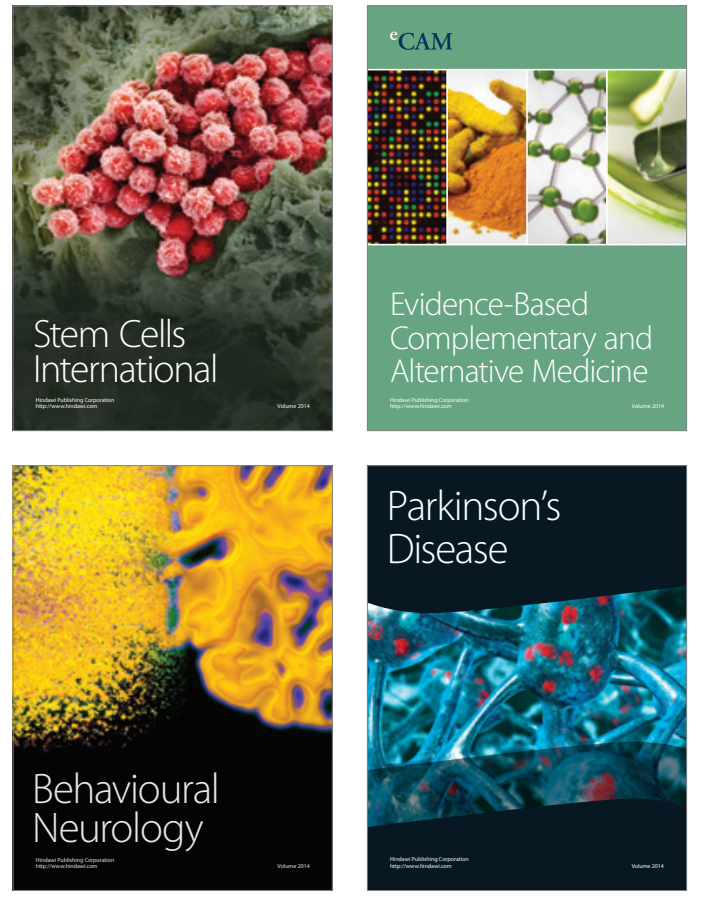
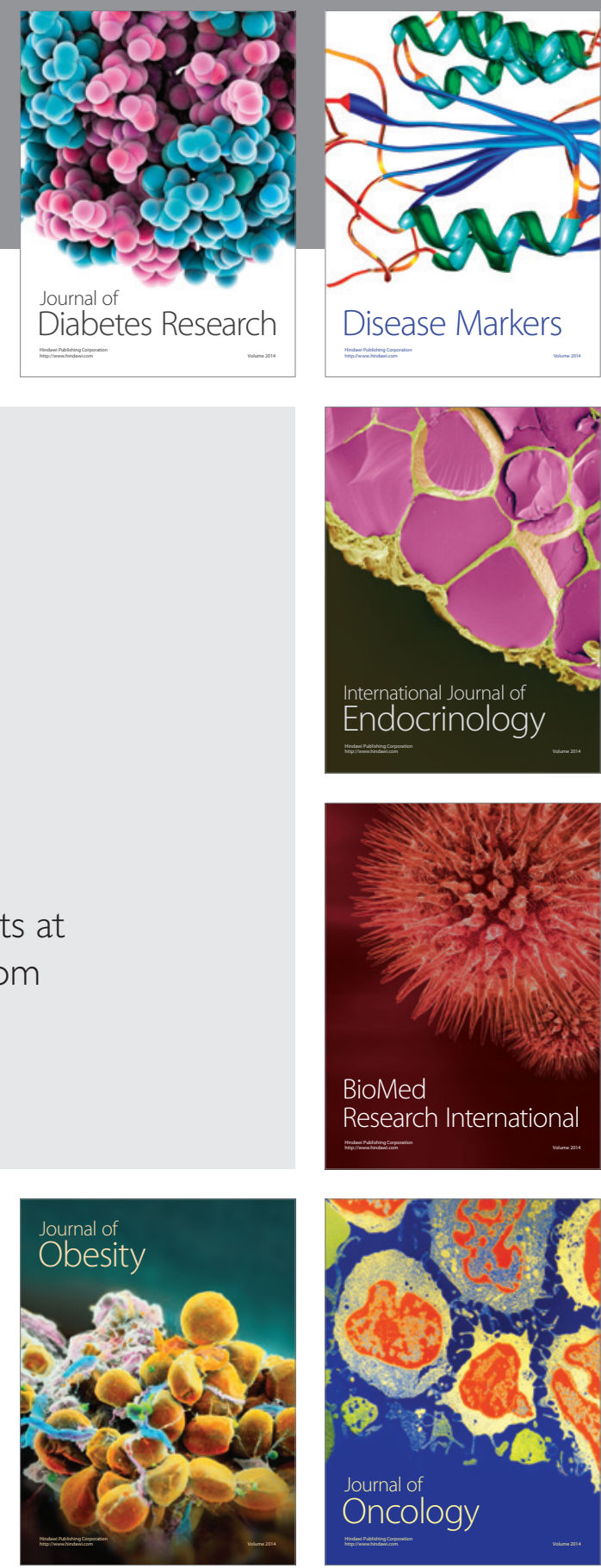

Disease Markers
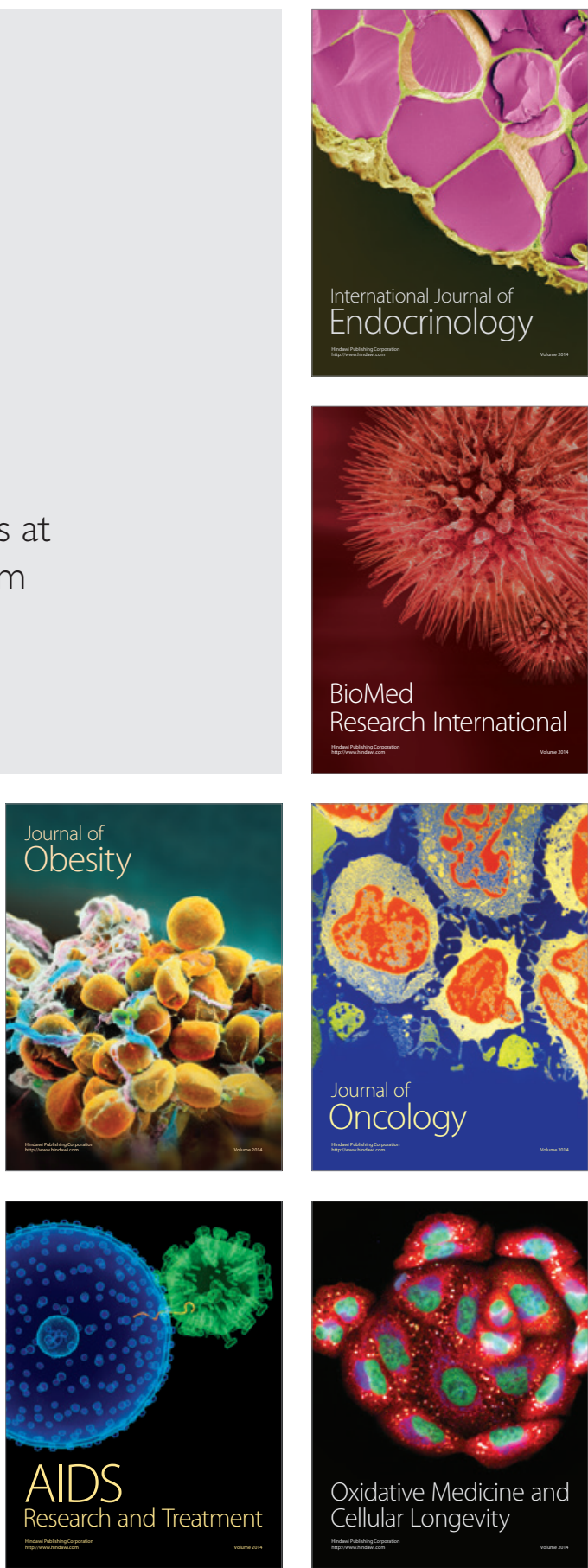